\title{
Research on the Mechanism of Radiation-Related Cognitive Impairment-A Review
}

\author{
He Zuo, Huaidong Cheng* \\ Department of Oncology, The Second Affiliated Hospital of Anhui Medical University, Hefei, China \\ Email: *chd1975ay@126.com
}

How to cite this paper: Zuo, H. and Cheng, H.D. (2019) Research on the Mechanism of Radiation-Related Cognitive Impairment-A Review. Journal of Cancer Therapy, 10, 188-196.

https://doi.org/10.4236/jct.2019.102015

Received: February 2, 2019

Accepted: February 19, 2019

Published: February 22, 2019

Copyright (อ 2019 by author(s) and Scientific Research Publishing Inc. This work is licensed under the Creative Commons Attribution International License (CC BY 4.0).

http://creativecommons.org/licenses/by/4.0/

\begin{abstract}
Radiotherapy (RT) remains a kind of front-line treatment for the control of tumor growth, especially for brain cancer. It is well known that cognitive impairment is mainly caused by cerebral RT, but the mechanisms underlying radiation-induced cognitive impairment remain unclear. There are different views on the cognitive dysfunction caused by RT in different studies. The recently research evidences about this topic are reviewed in present work. We found a few of factors including brain injury directly caused by radiation, cerebral tissue ischemia and necrosis caused by cerebrovascular injury, oxidative stress and brain injury mediated by inflammation, etc. which play a crucial role in the cognitive dysfunction caused by radiotherapy. These have all been widely recognized, and recently evidences have also shown that potential therapeutic interventions can effectively prevent cognitive impairment.
\end{abstract}

\section{Keywords}

Radiation, Cognitive Impairment, Cerebral

\section{Introduction}

According to statistics, the annual incidence about carcinoma of brain and neck in the world has been estimated at 1,240,000 [1]. At present, the methods for treating these tumors mainly include RT, surgery, chemotherapy, molecular targeted therapy, biological targeted therapy etc. [2]. As it is well known that RT is a kind of advanced cancer treatment, and between one-half and two-thirds of all newly diagnosed cancer patients need RT at some point during treatment [3]. $\mathrm{RT}$ is an important treatment modality for cancer patients, but various adjacent normal tissue toxicities may occur after RT [4], which may contribute to the ob- 
served cognitive dysfunction [5]. With the improvement of medical technology, the overall survival time of cancer patients is longer; the irradiation-induced cognitive impairment, including mild cognitive impairment to dementia, occurs in $50 \%-90 \%$ of patients with brain tumors who survive $>6$ months following irradiation [6] [7]. Because of radiation-induced cognitive impairment which significantly may affect their quality of life (QOL), now it is recognized as one of the most important outcome measurements, second only to survival in clinical trials [8], which becomes a most concerned problem in these patient and the practitioner [9].

The cognitive dysfunction caused by radiotherapy is widespread, mainly in the aspects of learning, work and life. Since the 1990s, it has been widely concerned by doctors. Although the mechanism of its occurrence is still unclear, studies have shown that cognitive impairment caused by radiotherapy is not impossible to prevent and treat, and potential therapeutic interventions can effectively prevent the occurrence of cognitive impairment [10], thus better improving the quality of life of cancer patients.

The mechanism of radiation-related cognitive impairment is less known, now the recently evidences on this topic are reviewed as follows.

\section{Definition of Radiation-Related Cognitive Impairment}

Radiation-related cognitive impairment is a symptom characterized by decline in wide domains of attention, memory, executive function, emotion, processing speed, and visual motor integration et al. [11] following radiotherapy. Cognitive impairment is now a significant risk factor for patients receiving conventional RT [12].

\section{Possible Mechanism of Radiation-Related Cognitive Impairment}

The radiation-related cognitive impairment most often results from radiation to the neck and brain. The mechanism of radiation-related cognitive impairment is still unclear, but studies have shown radiation-induced decline in cognitive function does not only result from an intractable change in brain structure [8]. Recently, in order to prevent and treat effectively on cognitive impairment caused by RT, some studies on the mechanism of cognitive impairment caused by RT were done.

Currently evidences generally suggested that the radiation-related cognitive impairment may be related to the following factors: 1) Radiation can cause the brain damage directly; 2) Cerebrovascular injury causes secondary cerebral tissue ischemia and necrosis; 3) Oxidative stress and inflammation mediate brain damage; 4) Other.

Radiation-induced brain injury in clinical RT is divided into the three types: acute (during up to days and weeks after irradiation), subacute or early-delayed (up to 12 weeks after irradiation) and late delayed (more than six months to 
years after irradiation). It is well-established that Single whole-brain irradiation with large-scale doses (2 - $10 \mathrm{~Gy})$ led to steep increases of apoptosis in the hippocampal DG within three to six hours after irradiation [12], because the hippocampus is an important brain area which is critical for the acquisition (learning), consolidation, and retrieval of declarative memories [13]. Studies have shown that radiation can cause a significant reduction in hippocampal volume [14]. Radiation-induced injuries to the hippocampus play an important role in compromising neurocognitive functioning for patients with brain tumors [15]. Numerous research evidences have shown that hippocampus injury is involved in cognitive impairment from RT [16]. The study evidences using radiation-induced learning and memory deficit in animal models have shown that RT can directly lead to apoptosis of hippocampal cells and further decrease hippocampal based cognitive function [17] [18]. In addition, some other studies have also shown that neurogenesis plays an important role in the pathogenesis of cognitive dysfunction after irradiation [19].

Although hippocampal injury is considered as one of the important causes of cognitive dysfunction caused by RT, some studies have found that non-hippocampal injury also can cause cognitive dysfunction, which may be related to acute and chronic vascular changes [20]. Recently results suggest that the brain white matter damage may not be a direct result of radiation but the more complicated interaction between fractionation, dose per fraction and ability of the tissue type to repair based on blood vessel density [21]. Accumulating evidence suggests that the capillary density will also be sparse when the dose of ionizing radiation is enough, and this may result in different degrees of cognitive impairment [22]. Cerebrovascular injury is one of the important mechanisms of radiation-related cognitive dysfunction.

Some other studies have shown that cognitive impairment caused by radiation may be related to microenvironmental factors such as oxidative stress and inflammation [6] [23]. As it is well-known that oxidative stress can result in the creation of reactive oxygen species (ROS) inside injured cells at a rate that overcomes cell repairing capability [24], which can lead to different level of brain damage. Radiation injury in the central nervous system (CNS) has also been linked to persistent microglial activation [25]. Neuroinflammation can activate microglia [22], cause brain white matter degeneration, necrosis and cerebrovascular injury, and cause all kinds of cognitive dysfunction [5]. Therefore, oxidative stress and inflammatory may be response for important mechanisms of radioactive brain injury.

To sum up, we found that the genesis of radiation induced cognitive decline is complex with multiple interacting and synergistic mechanisms [7]. A large number of studies have found that radiation-induced late side effects, including cognitive impairment, are hypothesized to occur due to dynamic interactions between multiple cell types within the brain, including astrocytes, endothelial cells, microglia, neurons and oligodendrocytes [8]. For example, maladaptive 
changes in astrocyte function might be implicated in radiation-induced cognitive dysfunction [26]. Other studies have shown that change in dendritic cells in neurons is also a factor in radioactive brain injury [27]. Therefore, previous evidences indicated that there are multiple factors on the radiation-induced cognitive impairment. All kinds of factors including sustained changes in hippocampal neurogenesis, loss of neuronal structure and synaptic plasticity, white matter damage, BBB damage, and reduced capillary density are associated with radiation-induced cognitive impairment [6]. Furthermore, recently there are also considerable evidence points to the depletion of neural stem and progenitor cells as one of the contributory mechanisms underlying radiation-induced cognitive dysfunction [28]. Studies have shown that oral administration of a pro-neurogenic compound exhibiting anti-inflammatory indications could impart long-term neurocognitive benefits in the irradiated brain [29]. And these mechanisms require further study to better understand the mechanisms associated with radiation-induced cognitive impairment.

\section{Factors Influencing Radiation-Related Cognitive Impairment}

The level of radiation-related cognitive impairment is influenced by multiple factors, such as the patient's age and the irradiated brain region may be the most important factors [22]. There is a large body of data showing that age is a risk factor for side effects of RT [30], and the effect on the developing adolescent brain is even more pronounced [31]. In addition, other studies have shown that exposure to different areas of the brain is not equally likely to cause the radiation-related cognitive impairment.

Cognitive impairment in patients with the bilateral hippocampal received RT is significantly worse than other brain regions, although the dose of radiation is the same [32].

Not only the age and exposure area are important factors for radiation-related cognitive impairment, but the dose and time of exposure are also important factors for radiation induced brain injury. Studies have shown that the cognitive dysfunction caused by radiation is dose-dependent, and a single exposure of 20 Gy is enough to cause cognitive dysfunction [4]. There is no doubt that radiation damage caused by high doses of radiation, but accumulating evidence suggests that various degrees of cognitive deficit can develop after much lower doses of ionizing radiation, as well [21]. A recent study showed that the longer the exposure, the greater the degree of brain damage [33]. The study also found a significant role for gender in radiation-induced cognitive impairment. The results suggested that adult males are more prone to radiation-related cognitive impairment than adult females, but there is no significant difference between adolescent males and females [34].

In addition to the above factors, therapy method is undoubtedly one of the important factors too. Recently studies have shown that the cognitive dysfunc- 
tion caused by micro-beam RT is less than that caused by broad-beam RT [35]. Clinical studies have also found that the cognitive impairment caused by particle therapy, which most commonly particle therapy, is much less than that caused by photon therapy (also known as X-ray therapy) [9]. As it is well-known that cognitive impairment termed chemobrain is a common neurotoxicity associated with chemotherapy treatment, affecting an estimated $78 \%$ of patients [36]. Therefore, the cognitive impairment caused by combination therapy is significantly increased when compared with RT alone [37]. In addition to these common factors, some other factors are also involved in the occurrence of radiation-related cognitive impairment. For example, the number of temporal cerebral microbleeds is also an independently associated with increased likelihood of cognitive dysfunction in patients with radiation-induced brain necrosis [38]. We also found that epigenetic mechanisms were a critical factor to radiation-related cognitive impairment, particularly for DNA methylation and histone modifications [39]. There are more and more evidences indicated that radiation-related cognitive impairment is caused by the synergistic effect of multiple influencing factors.

\section{Protect Radiation-Related Cognitive Impairment}

The study of the mechanism of radiation-related cognitive impairment is still not sure, and its potential interventions may be very complex. Previous evidences have shown that some potential methods can effectively reduce the occurrence of radiation damage. Stem cell transplantation is one of effective technique, some studies have shown that transplanting human stem cells into irradiated brains can solve the cognitive impairment caused by radiation [40] [41]. Perhaps in the near future, human stem cell transplantation will become an important measure to treat radiation-related cognitive dysfunction. Another study showed that oral administration of a pro-neurogenic compound exhibiting anti-inflammatory indications could impart long-term neurocognitive benefits in the irradiated brain, and then can reduce radiation damage [29].

It is well known that AT1R block can reduce the inflammatory response after cerebral ischemia and reduce the injury after cerebral ischemia [10]. Therefore, brain RAS inhibitors are attractive candidates for the treatment of radiation-induced normal tissue damage. Experiments have shown that treatment with RAS inhibitors, such as $1-158,809$, prevents or improves progressive cognitive impairment long after the completion of radiotherapy before, during, and after fractional whole-brain irradiation [42]. In addition to RAS inhibitors, studies have also found that the use of peroxisomal proliferation-activated receptor (PPAR) agonists such as pioglitazone before, during, and 4 weeks after treatment can prevent cognitive impairment [6].

In addition to these measures, studies have found that lithium treatment could ameliorate irradiation-induced so called late effects, such as cognitive decline and endocrine dysfunction, by preventing cell death and promoting regenera- 
tion [30]. The application of electroacupuncture immediately after brain irradiation can prevent the cognitive impairment caused by early radiotherapy, which is also a new way to prevent or improve the cognitive impairment of brain tumor patients requiring radiotherapy [6]. Others, such as targeting CCR 2 signals, may provide an effective way to reduce or prevent the incidence and severity of severe side effects such as ionizing radiation [43].

Although there are many methods to reduce the cognitive impairment caused by radiotherapy, specific measures that can be used in clinical practice are still very rare, and further advanced research is expected.

\section{Summary}

All in all, there are plenty of evidence on radiation-related cognitive impairment when all kinds of brain cancer patients following radiotherapy. Recently, the cognitive dysfunction related to RT has become an important factor to affect the long-term quality of life of brain cancer patients. RT related cognitive dysfunction has been widely concerned, but relatively few studies have been conducted on its specific mechanism. Although studies have shown that potential therapeutic interventions can effectively prevent/mitigate radiotherapy injuries, there are few specific clinical applications. We hope that there are more and more relevant research evidence in the future, and that radiation-related cognitive impairment in these patients can be found earlier, so that these patients' quality of life may be improved.

\section{Funding}

This research was supported by the National Natural Science Foundation of China (No. 81872504).

\section{Conflicts of Interest}

The authors declare no conflicts of interest regarding the publication of this paper.

\section{References}

[1] Ferlay, J., et al. (2015) Cancer Incidence and Mortality Worldwide: Sources, Methods and Major Patterns in GLOBOCAN 2012. International Journal of Cancer, 136, E359-E386.

[2] Patel, J.D., et al. (2014) Clinical Cancer Advances 2013: Annual Report on Progress Against Cancer from the American Society of Clinical Oncology. Journal of Clinical Oncology, 32, 129-160.

[3] Feiock, C., et al. (2016) Central Nervous System Injury-A Newly Observed Bystander Effect of Radiation. PLOS ONE, 11, e0163233.

[4] Zhang, L.Y., et al. (2012) Effects of Expression Level of DNA Repair-Related Genes Involved in the NHEJ Pathway on Radiation-Induced Cognitive Impairment. Journal of Radiation Research, 54, 235-242.

[5] Andrews, R.N., et al. (2017) Cerebrovascular Remodeling and Neuroinflammation 
Is a Late Effect of Radiation-Induced Brain Injury in Non-Human Primates. Radiation Research, 187, 599.

[6] Fan, X.-W., et al. (2014) Electroacupuncture Prevents Cognitive Impairments by Regulating the Early Changes after Brain Irradiation in Rats. PLOS ONE, 10, e0122087.

[7] Makale, M.T., et al. (2017) Brain Irradiation and Long-Term Cognitive Disability: Current Concepts.

[8] Dana, G.S., Elizabeth, M. and Robbins, M.E. (2013) Molecular Pathways: Radiation-Induced Cognitive Impairment. Clinical Cancer Research, 19, 2294-2300.

[9] Grosshans, D.R., et al. (2017) The Role of Image-Guided Intensity Modulated Proton Therapy in Glioma. Neuro-Oncology, 19, ii30-ii37.

[10] Duman, J.G., et al. (2017) Memantine Prevents Acute Radiation-Induced Toxicities at Hippocampal Excitatory Synapses. Neuro-Oncology, 20, 655-665.

[11] Castellino, S.M., et al. (2014) Developing Interventions for Cancer-Related Cognitive Dysfunction in Childhood Cancer Survivors. Journal of the National Cancer Institute, 106, 2-10.

[12] Balentova, S. and Adamkov, M. (2015) Molecular, Cellular and Functional Effects of Radiation-Induced Brain Injury: A Review. International Journal of Molecular Sciences, 16, 27796-27815.

[13] Acharya, M.M., et al. (2015) Human Neural Stem Cell Transplantation Provides Long-Term Restoration of Neuronal Plasticity in the Irradiated Hippocampus. Cell Transplatation, 24, 691-702.

[14] Karunamuni, R.A., et al. (2017) Abnormalities in Hippocampal Volume of Glioma Patients Prior to Radiotherapy. Acta Oncologica, 56, 427-430.

[15] Uto, M., et al. (2016) Non-Coplanar Volumetric-Modulated Arc Therapy (VMAT) for Craniopharyngiomas Reduces Radiation Doses to the Bilateral Hippocampus: a Planning Study Comparing Dynamic Conformal Arc Therapy, Coplanar VMAT, and Non-Coplanar VMAT. Radiation Oncology, 11, 1-8.

[16] Armstrong, C.L., et al. (2012) Phase Measurement of Cognitive Impairment Specific to Radiotherapy. International Journal of Radiation Oncology, Biology, Physics, 83, e319-e324.

[17] Zhang, L., et al. (2014) Minocycline Ameliorates Cognitive Impairment Induced by Whole-Brain Irradiation: An Animal Study. Radiation Oncology, 9, 281.

[18] Xu, M., et al. (2017) NFAT3/c4-Mediated Excitotoxicity in Hippocampal Apoptosis DURING Radiation-Induced Brain Injury. Journal of Radiation Research, 58, 827-833.

[19] Ding, X., et al. (2017) The p75 Neurotrophin Receptor Regulates Cranial Irradiation-Induced Hippocampus-Dependent Cognitive Dysfunction. Oncotarget, 8, 40544-40557.

[20] Hanbury, D.B., et al. (2015) Pathology of Fractionated Whole-Brain Irradiation in Rhesus Monkeys (Macaca mulatta). Radiation Research, 183, 367.

[21] Peiffer, A.M., et al. (2014) Radiation-Induced Cognitive Impairment and Altered Diffusion Tensor Imaging in a Juvenile Rat Model of Cranial Radiotherapy. International Journal of Radiation Biology, 90, 799-806.

[22] Lumniczky, K., Szatmári, T. and Sáfrány, G.J.F.I.I. (2017) Ionizing Radiation-Induced Immune and Inflammatory Reactions in the Brain. Frontiers in Immunology, 8, 517.

[23] Acharya, M.M., Martirosian, V., Christie, L.A. and Limoli, C.L. (2014) Long-Term 
Cognitive Effects of Human Stem Cell Transplantation in the Irradiated Brain. International Journal of Radiation Biology, 90, 816.

[24] Russi, E.G., Raber-Durlacher, J.E. and Sonis, S.T.J.M.O.I. (2014) Local and Systemic Pathogenesis and Consequences of Regimen-Induced Inflammatory Responses in Patients with Head and Neck Cancer Receiving Chemoradiation. Mediators of Inflammation, 2014, Article ID: 518261.

[25] Acharya, M.M., et al. (2016) Elimination of Microglia Improves Cognitive Function Following Cranial Irradiation. Scientific Reports, 6, Article No. 31545.

[26] Acharya, M.M., et al. (2016) Adenosine Kinase Inhibition Protects against Cranial Radiation-Induced Cognitive Dysfunction. Frontiers in Molecular Neuroscience, $\mathbf{9}$, 42 .

[27] Cacao, E., Parihar, V.K., Limoli, C.L. and Cucinotta, F.A. (2018) Stochastic Modeling of Radiation-Induced Dendritic Damage on in Silico Mouse Hippocampal Neurons. Scientific Reports, 8, Article No. 5494.

[28] Spurlock, M.S., et al. (2017) Amelioration of Penetrating Ballistic-Like Brain Injury Induced Cognitive Deficits after Neuronal Differentiation of Transplanted Human Neural Stem Cells. Journal of Neurotrauma, 34, 1981-1995.

[29] Allen, B., et al. (2018) Remediation of Radiation-Induced Cognitive Dysfunction through Oral Administration of the Neuroprotective Compound NSI-189. Radiation Research, 189, 345-353.

[30] Forbes, M.E., Paitsel, M., Bourland, J.D. and Riddle, D.R. (2014) Early-Delayed, Radiation-Induced Cognitive Deficits in Adult Rats Are Heterogeneous and Age-Dependent. Radiation Research, 182, 60-71. https://doi.org/10.1667/RR13662.1

[31] Zhou, K., et al. (2017) Lithium Protects Hippocampal Progenitors, Cognitive Performance and Hypothalamus-Pituitary Function after Irradiation to the Juvenile Rat Brain. Oncotarget, 8, 34111-34127.

[32] Shi, L., et al. (2018) Radiation-Induced Gray Matter Atrophy in Patients with Nasopharyngeal Carcinoma after Intensity Modulated Radiotherapy: A MRI Magnetic Resonance Imaging Voxel-Based Morphometry Study. Quantitative Imaging in Medicine and Surgery, 8, 902-909. https://doi.org/10.21037/qims.2018.10.09

[33] Brzozek, C., Benke, K.K., Zeleke, B.M., Abramson, M.J. and Benke, G. (2018) Radiofrequency Electromagnetic Radiation and Memory Performance: Sources of Uncertainty in Epidemiological Cohort Studies. International Journal of Environmental Research and Public Health, 15, E592.

[34] Perez, E.C., et al. (2018) Olfactory Memory Impairment Differs by Sex in a Rodent Model of Pediatric Radiotherapy. Frontiers in Behavioral Neuroscience, 12, 158.

[35] Bazyar, S., et al. (2017) Neurocognitive Sparing of Desktop Microbeam Irradiation. Radiation Oncology, 12, 127.

[36] Chiu, G.S., et al. (2017) Pifithrin- $\mu$ Prevents Cisplatin-Induced Chemobrain by Preserving Neuronal Mitochondrial Function. Cancer Research, 77, 742-752.

[37] Mcginnis, G.J., et al. (2016) Neuroinflammatory and Cognitive Consequences of Combined Radiation and Immunotherapy in a Novel Preclinical Model. Oncotarget, 8, 9155-9173.

[38] Shen, Q., et al. (2015) Temporal Cerebral Microbleeds Are Associated with Radiation Necrosis and Cognitive Dysfunction in Patients Treated for Nasopharyngeal Carcinoma. International Journal of Radiation Oncology, Biology, Physics, 94, 1113-1120.

[39] Acharya, M.M., et al. (2017) Epigenetic Determinants of Space Radiation-Induced 
Cognitive Dysfunction. Scientific Reports, 7, Article No. 42885.

[40] Smith, S.M. and Limoli, C.L.J.C.S.C.R. (2017) Stem Cell Therapies for the Resolution of Radiation Injury to the Brain. Current Stem Cell Reports, 3, 342-347.

[41] Acharya, M.M., et al. (2015) Defining the Optimal Window for Cranial Transplantation of Human Induced Pluripotent Stem Cell-Derived Cells to Ameliorate Radiation-Induced Cognitive Impairment. Stem Cells Translational Medicine, 4, 74-83.

[42] Joseph, J.P., et al. (2014) The Angiotensin Type 2 Receptor Agonist Compound 21 Elicits Cerebroprotection in Endothelin-1 Induced Ischemic Stroke. Neuropharmacology, 81, 134-141.

[43] Karim, B., Jopson, T., Arellano, C., Fike, J.R. and Rosi, S. (2013) CCR2 Deficiency Prevents Neuronal Dysfunction and Cognitive Impairments Induced by Cranial Irradiation. Cancer Research, 73, 1201-1210. 